\title{
Characterizing the microenvironment surrounding protein sites
}

\author{
STEVEN C. BAGLEY AND RUSS B. ALTMAN \\ Section on Medical Informatics, Stanford University School of Medicine, MSOB X-215, Stanford, California 94305-5479
}

(RECEIVED November 4, 1994; ACCEPTED January 23, 1995)

\begin{abstract}
Sites are microenvironments within a biomolecular structure, distinguished by their structural or functional role. A site can be defined by a three-dimensional location and a local neighborhood around this location in which the structure or function exists. We have developed a computer system to facilitate structural analysis (both qualitative and quantitative) of biomolecular sites. Our system automatically examines the spatial distributions of biophysical and biochemical properties, and reports those regions within a site where the distribution of these properties differs significantly from control nonsites. The properties range from simple atom-based characteristics such as charge to polypeptide-based characteristics such as type of secondary structure. Our analysis of sites uses nonsites as controls, providing a baseline for the quantitative assessment of the significance of the features that are uncovered. In this paper, we use radial distributions of properties to study three well-known sites (the binding sites for calcium, the milieu of disulfide bridges, and the serine protease active site). We demonstrate that the system automatically finds many of the previously described features of these sites and augments these features with some new details. In some cases, we cannot confirm the statistical significance of previously reported features. Our results demonstrate that analysis of protein structure is sensitive to assumptions about background distributions, and that these distributions should be considered explicitly during structural analyses.
\end{abstract}

Keywords: biophysical properties; calcium binding; computational biology; disulfide bridges; microenvironment; protein structure analysis; serine proteases; software

Central to molecular biology is the determination of macromolecular structure and the analysis of how structural elements produce an observed function. The principles by which structure relates to function have been elucidated in a piecemeal fashion, from work on single structures or small classes of structures. Computational assistance has come primarily in the form of graphical methods for scientific visualization and from special purpose programs for analyzing individual biophysical properties (such as solvent accessibility or electrostatic fields). Unfortunately, studying structures individually entails a risk of missing important relationships that would be revealed by pooling relevant data. The expected surfeit of protein structures provides an opportunity to develop tools for automatically examining biological structures and producing useful representations of the key biophysical and biochemical features. The utility of a general purpose system for producing these representations would extend from medical/pharmaceutical applications (model-based drug design, comparing pharmacological

Reprint requests to: Russ B. Altman, Section on Medical Informatics, Stanford University School of Medicine, MSOB X-215, Stanford, California 94305-5479; e-mail: altman@camis.stanford.edu. activities) to industrial applications (understanding structural stability, protein engineering).

In this paper we describe a computational tool for analyzing protein sites - microenvironments within a structure distinguished by their structural or functional roles. We define a site as a region within a macromolecule with a central location and a surrounding neighborhood. In principal, a site could include the entire molecule, but we focus on sites that involve proper subsets of the molecule using a neighborhood with a 10 - $\AA$ radius. Sites can be significant because of their structural role (for example, the site where a disulfide bond forms), their functional role (the active site of a serine protease) or both (the site of calcium binding). The most basic representation of a site is the set of atoms within it, along with their three-dimensional coordinates. We have created a system that augments this representation with the spatial distribution of user-defined properties. These properties include labels designating the types of atoms, chemical groups, amino acids, and secondary structures. They also include simple biophysical characteristics such as charge, polarity, mobility, and solvent accessibility.

The distribution of a property is computed by dividing the total volume of a site into subvolumes and computing the prev- 
alence of the property within each of these subvolumes. Such distributions can be computed for sites, as well as for other microenvironments that are taken as nonsites. We have built the system on the assumptions that the key features of a microenvironment are defined with respect to a background distribution, and that the background distribution should be derived from the data, not from prior assumptions (such as spatial uniformity). The system therefore compares the distribution of the properties in sites (the positive examples) with the distribution of the same properties in user-specified nonsites (the negative examples, used as controls). Properties for which the site and nonsite distributions are different to a statistically significant degree are reported. These statistically interesting properties should be considered preliminary hypotheses that allow an investigator to focus attention on regions that may be responsible for the particular structure or function of interest. It may also find use in the testing and verification of predictions.

In this implementation, we have concentrated only on spherically symmetric, radial distributions (whereby the volume of a site is divided into concentric shell subvolumes) for three reasons. First, there are many structural sites that exhibit spherical symmetry, and these are useful test cases. Second, we would like to document that the principle of comparing sites with nonsites is an effective way to measure the significance of findings. Finally, we want to test the hypothesis that even sites with recognized nonradial asymmetries can profitably be analyzed with the weaker assumption of radial symmetry. To test these assumptions, we used the program to analyze three types of sites: calcium binding sites (known to have an approximately spherical symmetry) compared to randomly selected nonsites, sites surrounding disulfide bonds compared to nondisulfide cysteines, and the active site of the serine proteases (which exhibits a richer, nonspherical, spatial organization) compared to other sites with histidines. Because these sites have been extensively characterized in the literature, we are able to test the ability of the method to extract previously documented features of these sites. We also have an opportunity to report additional features, or dispute the significance of other features.

There are three problems that must be solved in order to effectively build tools for elucidating structure-function relationships. First, a good computational representation must be chosen that allows critical features to be manipulated in an interpretable and well-defined way. Property-based representations of macromolecular structure have been shown to facilitate the identification of key features. For example, Bowie et al. (1991) used a set of base properties (including secondary structure, degree of solvent accessibility, and polarity) to show that these higher level representations are useful for distinguishing proper from improper three-dimensional folds. Similarly, Zvelebil and Sternberg (1988) have shown that properties can be used to characterize the neighborhood of catalytic residues (properties included residue type, mobility, polarity, and sequence conservation).

The second problem is finding a good way to characterize the spatial distribution of critical properties. There have been numerous studies of specialized protein structural features that have been performed over a large set of structures (such as sulfuraromatic interactions (Reid et al., 1985), amino-aromatic interactions (Mitchell et al., 1994), ion pairs (Barlow \& Thornton, 1986), distribution of charge (Barlow \& Thornton, 1986), distribution of hydropathy (Korn \& Burnett, 1991), anion-ligand interactions (Chakrabarti, 1990a, 1990b, 1993, 1994), patterns of hydration (Roe \& Teeter, 1993), protein side-chain interactions (Singh \& Thornton [1992] and others). For each of these studies, a standard coordinate system (or frame of reference) was chosen in order to provide the most useful perspective on the data. One data structure for spatial distributions that has been demonstrated to be quite general is a three-dimensional grid, in which properties of an object or image can be stored and manipulated. For example, a three-dimensional grid has been used to study binding energies in the context of proteinligand interactions (Goodford, 1985). Grids offer the advantage of a global coordinate system, scalable resolution, and easy local search.

The third problem that must be solved in order to uncover important structural or functional features in macromolecules is that of determining significance. The structure of a single macromolecule provides so much information that it is often difficult to determine which aspects of the structure are critical, novel, or in some other way distinguished. Very often, the background distribution that is used to compare the observed distribution of features is assumed to be either spatially uniform, or computed from marginal distributions using an independence assumption. Then, a comparison between expected and observed feature distributions can yield some insight (although statistical significance does not guarantee biochemical importance). This criterion has been successfully applied in the study of atomic interactions (Warme \& Morgan, 1978), the study of some protein microenvironments (Reid et al., 1985; Singh \& Thornton, 1992; Walshaw \& Goodfellow, 1993), and sequence-structure correlations (Klingler \& Brutlag, 1993, 1994), and in constructing context-sensitive potential functions (Sippl, 1990; Rooman et al., 1992). There are, however, advantages to choosing background "control" distributions that are not parametric or assumed uniform but are computed from a population of negative examples of the feature of interest. For example, when studying the environment of calcium in proteins, the choice of control background could be the environment of other, noncalcium cations (e.g., magnesium or manganese), or it could be a general arbitrary background (any atom). In the first case, the analysis would highlight the details that might help proteins distinguish between cations (detailed geometry), whereas the second case would highlight the details that help proteins bind to cations in general (anions). Drawing together the concepts of propertybased representations, spatial distributions, and significance measured with respect to an explicit control group, we have built a system that is able to detect the features that distinguish sites from nonsites. This system is a new tool for use in the larger scientific endeavor of elucidating protein structure and function.

\section{Results}

As detailed in the Methods section, our grid-based system accumulates the spatial distribution of physical features (currently about 20, see Appendix 1) for protein microenvironments. We allow the user to specify control microenvironments that define the significant features of the test distribution. We use a standard nonparametric test of significance (Mann-Whitney ranksum) when comparing test and control distributions. We have built a general purpose system that makes such comparisons easy and not dependent on the microenvironment under study. For 
each of the three sites studied, we present an analysis of the features that are reported as significant by the program, along with a discussion of similar features reported in the literature. The Electronic Appendix contains the detailed significance measures, and a ranking of the significance of all findings for each of the three sites studied.

\section{$\mathrm{Ca}^{2+}$ binding sites}

Our results for the calcium binding sites are displayed in Figure 1 and Kinemage 1 . The key findings can be summarized upon inspection: there is a statistically significant excess of neg- atively charged, acidic, oxygen-rich, mostly Asp and Glu moieties at radii 2-7 $\AA$. Conversely, there is a relative paucity of hydrophobic (particularly Leu, Val, and Tyr), nonpolar moieties. These findings are consistent with the general understanding of cation binding sites, and particularly, the studies that have noted that metal sites in proteins are commonly surrounded by an inner shell of hydrophilic ligands and outer shell of carboncontaining groups (Yamashita et al., 1990; Nayal \& Di Cera, 1994). The program produced other significant findings:

1. The property any-atom shows a sparsely occupied shell at 1-2 $\AA$ (indicating empty space) and relatively concentrated shells at 2-5 (indicating tightly packed atoms). The shells $0-3 \AA$ are deficient in carbons, $2-3 \AA$ is deficient in nitrogens, and 2-3,

\section{Calcium Binding Site}

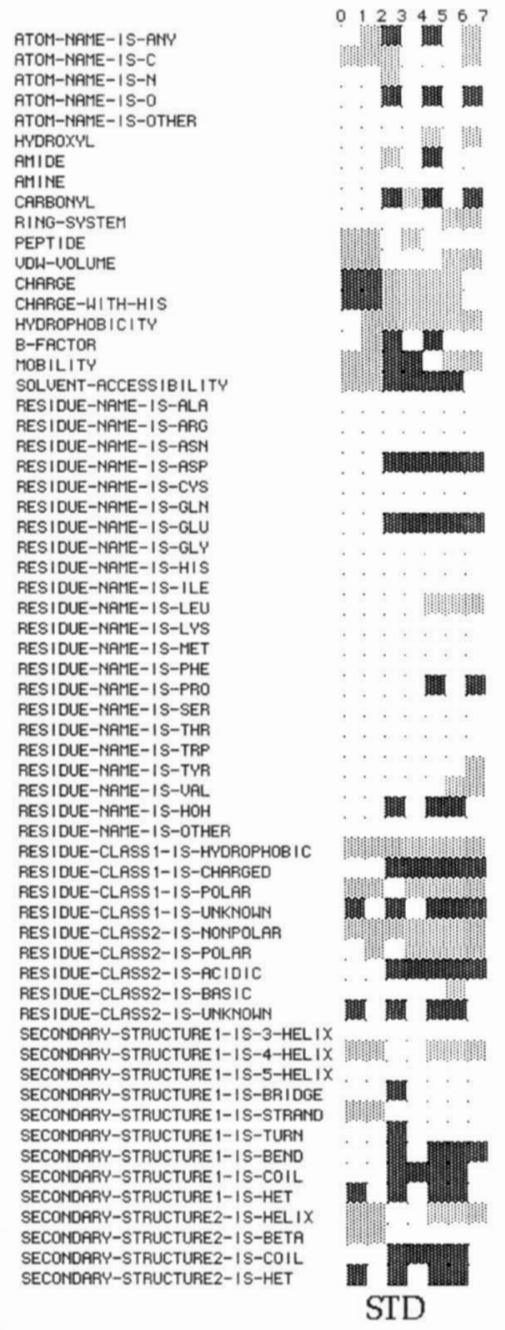

ATOM-NAME-IS-ANY
ATOM-NRME-IS-C

ATOM-NAME-1S-C

ATOM-NAME-IS-0

RING-SYST

CHRRGE

FACTOR

SOLUENT-ACCESSIBILITY

RESIDUE-NAME-IS-ALA

DUE-NAME-IS-ASN

RESIDUE-NAME-IS-CYS

ESIDUE-NAME-IS-GLN

ESIDUE-MAME-IS-GLY

RESIDUE-NAME-1S-HIS

AESTIDE-CLSS-IS-POLAR

ECONDOPY-STRUCTURE $1-15-4-H E L I X$

ECONORRY-STRUCTURE $1-15-5-$ HEL

SECONORRY-STRUCTURE -IS-BRIDGE

DUCTURE-IS-HELIX

ECONDARY-STR

STD

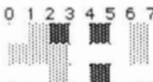

II.

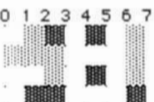

斯

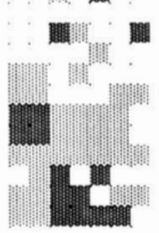

頙
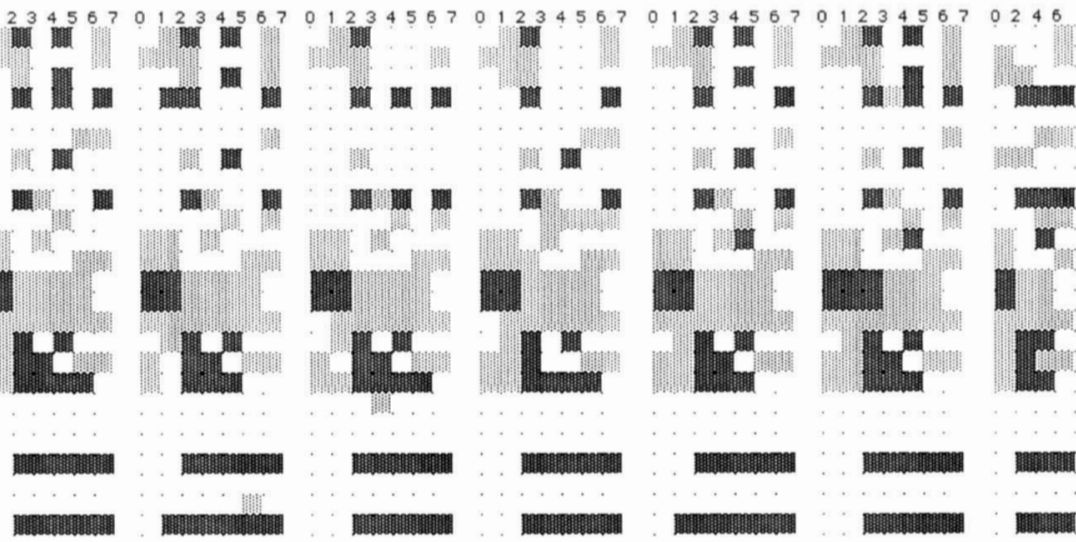

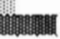

Hal

閴
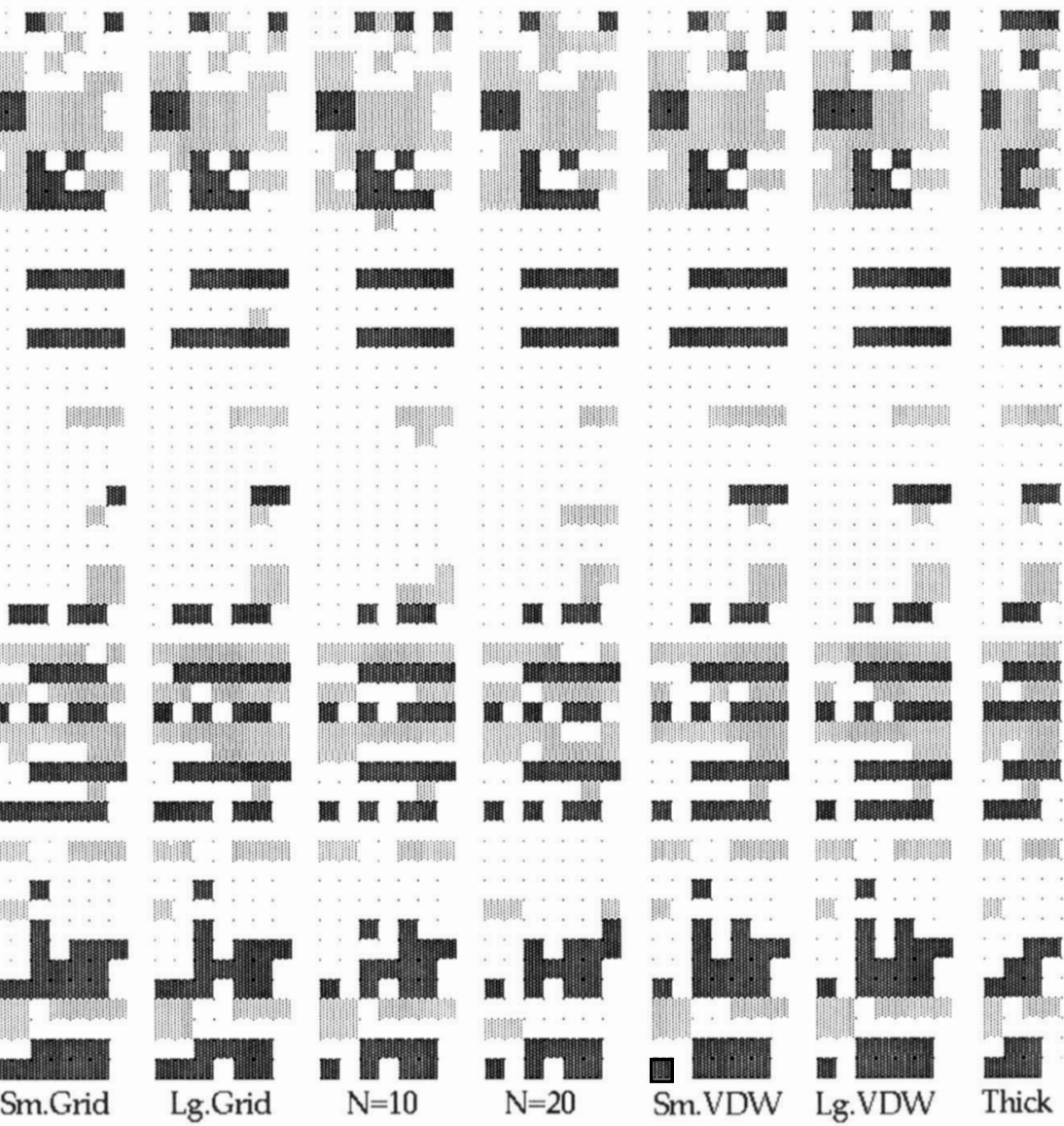

Herte
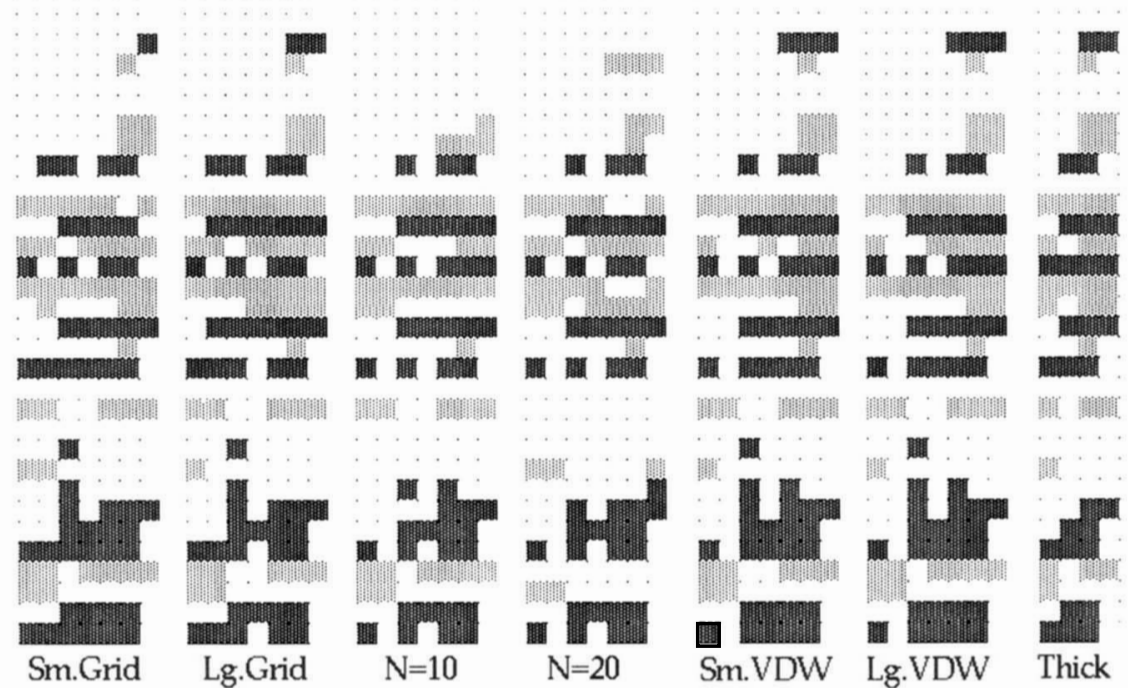

Fig. 1. Results for the $\mathrm{Ca}^{2+}$ binding sites showing significant properties and shells. Output is displayed as a two-dimensional array, with the significant results marked as nonwhite cells. Properties fall along the vertical axis; the shell volumes along the horizontal axis (for example, shell 0 is the shell from 0 to $1 \AA$ around the origin). Dark gray cells mark property/volume pairs for which the site value was greater than the controls; the light gray marks cells for which the site value was less than the controls. Results of eight experiments described in the text are summarized in the columns: (1) the baseline calculation with $P<$ 0.01 (STD); (2) the smaller grid (Sm.Grid); (3) the larger grid (Lg.Grid); (4) a nonsite sample size of $10(N=10) ;(5)$ a resample of 20 nonsites $(N=20)$; (6) a smaller van der Waals radius (Sm.VDW); (7) and larger van der Waals radius (Lg.VDW); and (8) collection of shells with thickness of $2 \AA$, instead of $1 \AA$ (Thick). 
4-5, and 6-7 $\AA$ have a greater than expected concentration of oxygen. This distribution is as expected given the spacing of the coordinating oxygen shells and their van der Waals radii. The results of Yamashita et al. (1990) and Nayal and Di Cera (1994) suggest that the oxygen shell around a calcium ion is surrounded by a larger shell of nitrogen. There is a nitrogen shell at 4-5 significant at $P<0.02$, just below the threshold for the other results reported here.

2. The amide group is underrepresented in shells $2-3$ and overrepresented in shells $4-5$. The oxygen-supplying carbonyl groups are strongly represented in the shell $2-3,4-5$, and 6-7, similar to the oxygen distribution seen at the atomic level.

3. There is a significant deficit of ring-system atoms in the shells 5-7. As the ring systems are hydrophobic, it follows that they would avoid the center of the charged $\mathrm{Ca}^{2+}$-binding neighborhood. There is a deficit of ring-system atoms in the inner shells ( $0-4)$ as well, but at a lower level of significance (see Electronic Appendix).

4. The peptide property shows that the shells near the calcium are not filled with peptide (backbone) atoms, which is appropriate given the greater ability of side groups to supply chargecarrying oxygen atoms. The peaks of the $B$-factor in shells $2-5$ show the relatively high crystallographic disorder, likely due to the presence of the side chain atoms (mostly oxygen) at that distance (which also correlates with the relative absence of peptide atoms near the $\mathrm{Ca}^{2+}$ ). There is greater mobility in the atoms in shells $2-5$, consistent with the observation of excess side-chain atoms.

5. As expected, charge shows a significant distribution, with the inner two shells containing more (more positive) charge, due to the calcium ion, and the surrounding shells holding less (more negative) charge, because of the coordinating oxygens. Also as expected, the hydrophobicity property shows that the shells surrounding calcium are not hydrophobic. Solvent accessibility, which peaks in the range of $2-5$, reflects the relatively short distance of the site from the protein surface.

6 . In the residue property distributions, the residues Asp and Glu are very strongly represented in the shells $2-7$, reflecting their role as a primary source of oxygen. Water is also strongly represented in shells $2-3$, and $4-6$, as it also can provide a coordinating oxygen. Nayal and Di Cera (1994) note the presence of waters, but disregard them in their valence computation.

7. The hydrophobic Leu and Val are underrepresented, as is Tyr in one shell 5-6. Pro is overrepresented in shells 4-7. To our knowledge, these observations have not been previously noted. The two-residue class systems show expected distributions: more charged residues and fewer polar or hydrophobic residues. The acidic class captures the presence of Asp and Glu. The unknown class is a catch-all; in this case, it shows the overrepresentation of calcium at the center and waters in the surrounding environment.

8. The secondary structure findings show a significant relative absence of helices and abundance of coils. The heterogeneous groups found nearby (secondary-structure-is-het) are calcium at the center and water molecules further out.

The features identified here are consistent with the detailed analysis of metal ion binding sites (Sekharuda \& Sundaralingam, 1988; Chakrabarti, 1990a; Yamashita et al., 1990; Jernigan et al., 1994; Nayal \& Di Cera, 1994), except that the radial averaging loses information about the precise orientation of the coordinating groups with respect to the cation.

\section{Cys bonding sites}

Our property grid results for the Cys disulfide environment versus Cys nonbonding environments are displayed in Figure 2 and Kinemage 2. The key features of the neighborhood surrounding a cysteine that participates in a disulfide bond is the occurrence of a neighboring cysteine at 2-6 $\AA$, and this is a trivial consequence of our experimental setup. The other key features of the disulfide environment are an abundance of Tyr and a relative paucity of His and Ile. Muskal et al. (1990) noted the abundance of Tyr and attributed it to its hybrid hydrophobic/ polar character. There is a relative lack of helical residues in the neighborhood and an increase in polar, especially hydroxyl moieties, also reported in Fiser et al. (1992). More detailed observations and comparisons can also be made:

1. The local hydrophobicity is low in the shells at 0-3 A surrounding the disulfide bond. Recently, Karlin et al. (1994), in a study of the association of different residue types, suggest that when lone cysteines are studied separately from disulfide and metal-coordinating cysteines, they would exhibit hydrophobic characteristics, as is indeed observed here.

2 . The $B$-factor for the neighborhood of the disulfide sulfur is lower than the controls. This is expected, because disulfide bonds tend to stabilize structure overall.

3. Although we observe the polar effect (increased polar atoms at 1-3 $\AA$ ), as reported by Muskal et al. (1990), the other effects (preference for turns and bends, and charged residues) are not seen. In addition, the results of Fiser et al. (1992) (polar, neutral, and $\mathrm{H}$-bond-forming residues preferring bridges, and hydrophobic residues preferring free cysteines, Gly near half cystines) were not seen.

4. Muskal et al. (1990) trained a neural net on 128 cysteinecontaining protein structures using local sequence data (within five residues in either direction, centered on Cys) to predict the disulfide bonding state of the Cys. The predictions were about $80 \%$ accurate. Analysis showed relationships between bonding state and secondary structure, with disulfide environments preferring extended conformations, and nonbonding cysteines showing no preference. We detect only a small preference for helical structures among nonbonded cysteines (and address this further in our discussion of the sensitivity analysis).

5. Fiser et al. (1992) also commented that Gly was seen frequently in the sequence neighborhood around nondisulfide cysteines. At our significance level, we do not see them in the structural neighborhood of $10 \AA$, so their effects may be indirect (for example, because of segmental flexibility of the peptide chain).

\section{Serine protease active sites}

The enzymatic activity of proteins in the serine protease family is due, in part, to the three-dimensional positioning of the residues His, Asp, and Ser, forming the catalytic triad (Warshel et al., 1989; Greer, 1990; Branden \& Tooze, 1991; Perutz, 1992; Zhou et al., 1994). The active site is not spherically symmetric; we included it to test the sensitivity of the property search in the case - presumably common - when spherical symmetry is violated. The results are shown in Figure 3 and Kinemage 3. Not surprisingly, with a coordinate system centered around His and with other (nonactive site) His as controls, the program found that Asp occurs at 7-8 $\AA$ and Ser at 3-4 $\AA$ in the protease sites. In addition, an abundance of Cys were seen at 5-8 $\AA$, part of 


\section{Disulfide Bridge}
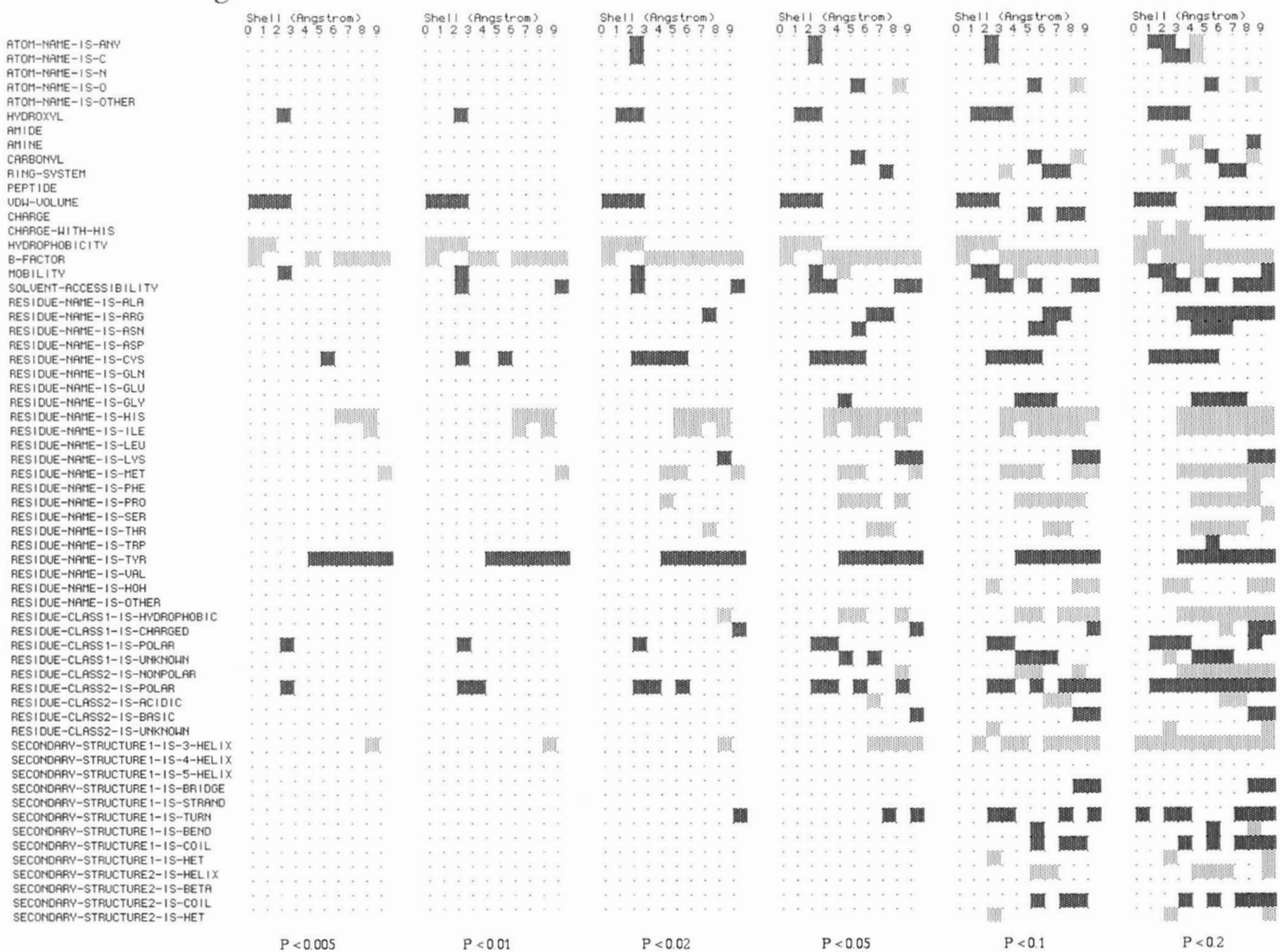

Fig. 2. Results for the disulfide bridge environment (in the context of lone cysteines) showing the significant properties and shells. This plot follows the same conventions as Figure 1. Results of varying the significance threshold are shown with significance values chosen from 0.005 to 0.2 . As described in the text, some findings reported in the literature only appear at low levels of significance.

a regularly recurring disulfide bond near the site. Other significant findings are:

1. There is a notable presence of helical elements in the active site. There is a very significant range of shells containing atoms forming 3-helices ( 0 -7 $\AA$ ).

2. The active site also shows greater solvent accessibility in the immediate neighborhood of the His (shells 0-3) compared to nonactive-site His residues, which is expected given the requirement that the active site be accessible to the molecule that is the object of the proteolytic action.

3. Shell 6-7 is deficient in carbonyl group atoms, whereas shell 8-9 shows a surfeit. This observation persists with a change of control group, suggesting that it is real. It correlates with the location of helical backbone segments at this radius.

\section{Sensitivity analysis}

We tested the sensitivity of the method to various modeling choices made in the implementation, as discussed in the Meth- ods section. The results of the sensitivity analyses are quite encouraging. The first four rows of Table 1 show that the method is not particularly sensitive to the grid spacing or the precise van der Waals radii used. In each case, $94 \%$ of the findings remain the same. Table 1 also demonstrates that reducing the sample size by $50 \%$ does not produce marked changes in the features that are reported as significant. Similarly, a total resampling of the nonsites leaves more than $90 \%$ of the features unchanged. The final column of Figure 1 demonstrates that the choice of shell thickness does not change the pattern of significant parameters. More importantly, none of these experiments show large changes in the magnitude of a finding, indicating a fair amount of stability of the method to differences in sampling.

As expected, the method does show sensitivity to the choice of nonsite controls. When we analyze the serine protease site centered on random atoms, instead of nonprotease His, we find that the presence of the His in the site becomes significant. In the case when we use nonprotease His, this property was controlled and so did not clutter the output. In general, the control 
Serine Protease Active Site

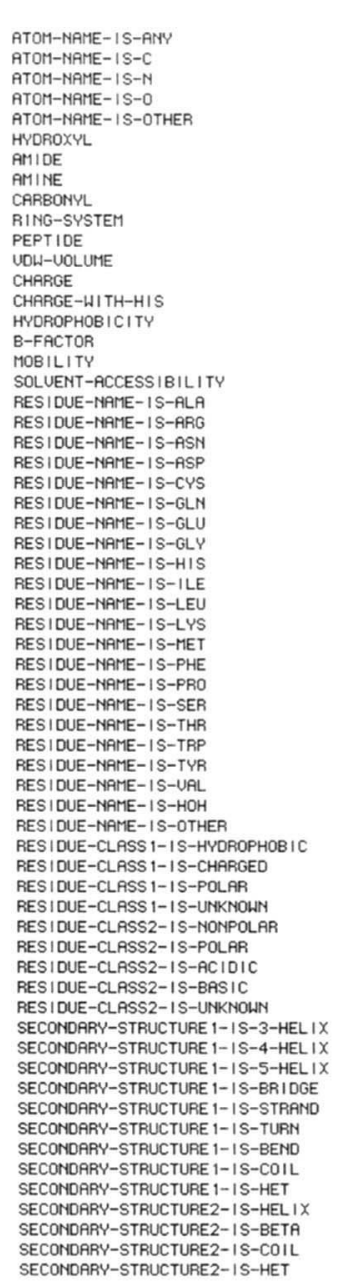

SECONOARY-STRUCTURE2-IS-HET
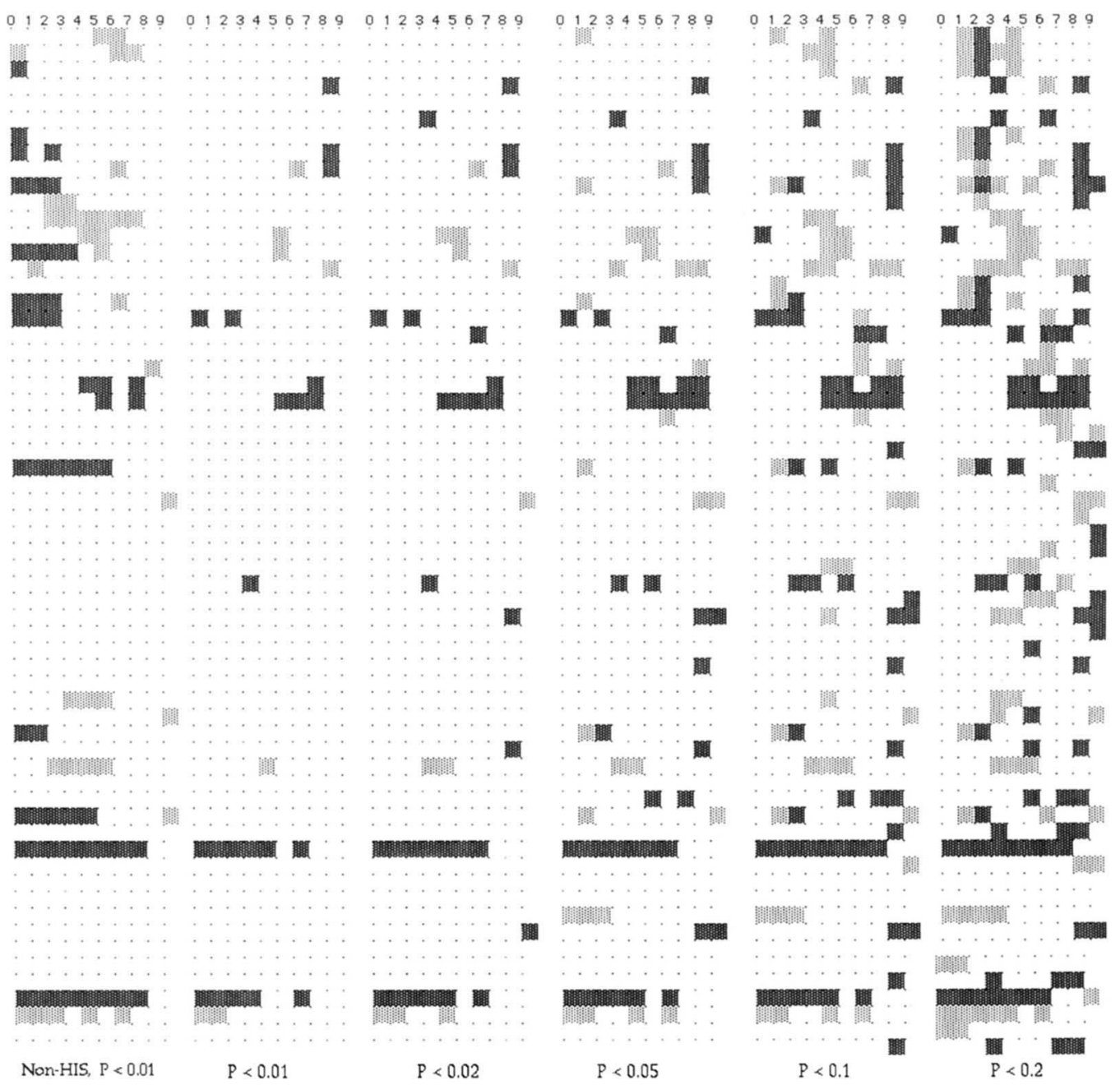

Fig. 3. Results for the serine protease active site showing the significant properties and shells. This plot follows the same conventions as Figure 1. The first column shows the features that are significant when the nonsite controls are chosen randomly. The second through sixth columns show features that are significant when the nonsite controls are nonactive-site histidines, with decreasing levels of significance from $P<0.01$ to $P<0.2$.

can be chosen so that spurious findings are de-emphasized. This principle also applies to our disulfide bridge analysis, in which the control sites are lone Cys residues. The analysis here stresses the differences between Cys in a disulfide environment and that in a nondisulfide environment. The findings may be very different from those that would be found in a general comparison of Cys environments versus other residue environments. Such an analysis would allow the relative environments of different residues to be studied in a systematic manner.

Finally, in order to test the sensitivity of our method to the definition of significance, we varied the cutoff for reporting significant differences between site and nonsite environments. We report the results of varying the threshold on both the disulfide and serine protease environments, for which some of previously described features (as detailed above), were not seen at our significance threshold. The significance cutoff for the results reported above was $P<0.01$. Figures 2 and 3 show the significant properties for cutoffs ranging from 0.005 to 0.2 . Not surpris- ingly, as the threshold is raised, the number of results reported as significant rises. This spectrum allows a more detailed comparison of our results with those reported in the literature. In several cases, we detected the previously reported results only at relatively low levels of significance. These include the preference of bonded Cys for turns, bends, and charged residues (Muskal et al., 1990), and for polar, and H-bond-forming residues (Fiser et al., 1992). Nonpolar residues were associated with nonbonded Cys at lower levels of significance. Also detected were increased frequency of Gly near half bridges but only at lower levels of significance $(P<0.05)$. In addition, the range of distances for which helices are present around nonbonded cysteines increases at lower thresholds. The preference of the disulfide bridge for extended conformations $(\beta)$ as noted in Muskal et al. (1990) was not detected at any level of significance. Our sensitivity analysis illustrates the value of using significance values grounded in statistical theory, which quantify the confidence with which distinctions can be made. 
Table 1. Summary of sensitivity analyses ${ }^{\mathrm{a}}$

\begin{tabular}{|c|c|c|c|c|c|c|c|c|}
\hline & \multicolumn{2}{|c|}{$\begin{array}{c}\text { Became } \\
\text { insignificant }\end{array}$} & \multicolumn{2}{|c|}{$\begin{array}{c}\text { Reversed } \\
\text { significance }\end{array}$} & \multicolumn{2}{|c|}{$\begin{array}{c}\text { Became } \\
\text { significant }\end{array}$} & \multicolumn{2}{|c|}{$\begin{array}{c}\text { No } \\
\text { change }\end{array}$} \\
\hline & $N$ & $\%$ & $N$ & $\%$ & $N$ & $\%$ & $N$ & $\%$ \\
\hline Small grid & 10 & 2.3 & 0 & 0.0 & 14 & 3.2 & 410 & 94.5 \\
\hline Big grid & 11 & 2.5 & 0 & 0.0 & 16 & 3.7 & 407 & 93.8 \\
\hline Big VDW & 11 & 2.5 & 0 & 0.0 & 13 & 3.1 & 410 & 94.5 \\
\hline Small VDW & 9 & 2.1 & 2 & 0.5 & 14 & 3.3 & 409 & 94.2 \\
\hline Smaller sample & 19 & 4.4 & 0 & 0.0 & 7 & 1.7 & 408 & 94.0 \\
\hline Resample & 26 & 6.0 & 0 & 0.0 & 12 & 2.9 & 396 & 91.2 \\
\hline Change control & 6 & 1.0 & 0 & 0.0 & 64 & 10.4 & 550 & 88.7 \\
\hline
\end{tabular}

\begin{abstract}
${ }^{a}$ For each experiment listed in a row, the changes to the number of property/volume pairs is shown. The reference for all comparisons is the results with a significance threshold of $P<0.01$. For each new property plot (such as those shown in Figs. 1, 2, 3), we compared the individual boxes within the array with the reference plot. We counted which boxes became insignificant in the new experiment, reversed significance, became significant, or had no change. The first six rows correspond to columns two through seven in Figure 1. The last row (Change control) refers to the experiment in changing control nonsites for serine proteases and corresponds to the first column of Figure 3.
\end{abstract}

\section{Discussion}

The most significant biological results of our experiments are contained in the plots of significance shown in Figures 1, 2, and 3. During the evolution of a site, the selective pressure is on the ability of the molecule to create an effective milieu (for the desired structure or function). The amino acids provide a basis set of chemical groups that can contribute certain characteristics to the site, but many characteristics can be realized in multiple ways. By temporarily abandoning a view of sites as groups of amino acids, and instead concentrating on the chemical milieu in important locations, we may gain insight into the critical factors that define the site. In essence, the plots shown in Figures 1, 2 , and 3 provide a new way of looking at these sites for the purpose of trying to understand their structure and function. In the case of the calcium binding site, the most interesting subtle features are those at the atomic and chemical group level. We see a periodicity in the occurrence of carbonyl groups at 2-3, 4-5, and 6-7 Ångstroms that persists throughout our sensitivity analysis. We also see a lack of amide nitrogens between 2 and $3 \AA$, but an abundance of amide nitrogens between 4 and $5 \AA$. These findings suggest subtle constraints on the structure of these sites that may be important for protein engineering applications. Although these correlations cannot be used to establish mechanism, they are significant enough over a large range of sites and nonsites to warrant attention. In general, the atom-based and chemical group-based properties provide the insight that is most likely to be functionally relevant. As currently implemented, our method does not correlate properties with protein sequence. The analysis of structure-sequence relationships is already the subject of intense interest. Our method is designed to look at the environment around a site without regard to the connectivity of participating residues. (Connectivity may be an interesting property to consider for further implementations.)

The residue and secondary structure-based properties are useful, however, for understanding the ways in which evolution has created these sites using its generic tools. It is not immediately obvious that construction of calcium binding sites would be characterized by residues that arise from coils and bends, but that serine protease active sites would be dominated by residues that arise from helical elements. Nevertheless, these observations are clear and consistent in our experiments. Although individual exceptions to these aggregate observations can be found, the weight of evidence toward certain types of structural components may be useful for engineering applications as well as for understanding functional roles.

There are several important issues related to the assessment and evaluation of our results and the method in general. Central to our method is the explicit use of a control group as the baseline for the statistical testing. The prominence of the control group in the calculation suggests that care should be taken in choosing it. This choice falls on the investigator, not the program itself. To return to an example mentioned earlier, the calcium binding sites can be compared to randomly chosen atoms (to find general properties of the binding site), or compared to other metal ion binding sites (to find specific properties that bind calcium, but not other metal ions). The data set, which is a sample from a larger population, should be representative of the population, so that significant properties reported for the sample apply more generally to the entire population. Particular care must be taken when the proteins have a close evolutionary relationship to avoid assigning undue importance to common but nonfunctional patterns. In some cases, the proper choice of the control group from the population may correct for this defect.

Our major goal in choosing the data sets (both experimental and control) for three sites was assessment. One difficulty in evaluating our method is that there is no independent, quantitative measure for accuracy for these experiments. The best qualitative assessment is a direct comparison with the results reported in the literature. To facilitate this comparison, we used a subset of the proteins upon which the original studies were based.

For the calcium binding and disulfide bridges, we had sample sizes for sites of 6 and 16, and for nonsites of 19 and 100 . Therefore, we have good statistical power for detecting significant differences. Of course, our results are affected by the selection of proteins used. The PDB has a preponderance of 
small to medium-sized globular proteins, with an overrepresentation of enzymes. Thus, if we used every example of each site, we would have a somewhat biased sample. Instead, we did not use every available site, but an arbitrary sampling of sites from among the alternatives. Strictly speaking, therefore, our results only apply to the sites listed in Table 2 . However, as detailed in the Results section, most of the findings in these two systems are either previously documented or biochemically reasonable and easily interpreted. In essence, the plots shown in Figures 1, 2 , and 3 are fingerprints for the features common to all these sites. The serine protease results, however, are limited by two factors. First, serine protease sites have important nonradial asymmetries that have been shown to be critical for function, such as the planar orientation of the three critical residues. Second, we have a relatively small sample size of serine proteases in the protein data bank. Our significance tests are sensitive to sample size and so require large magnitude differences in order to report findings at a significance of $P<0.01$. For these two reasons, our findings on the serine protease sites are rather sparse.

A related issue is the meaning of statistical significance. Just as the program does not choose the data sets used as input, it cannot assign scientific importance to the output. Statistical significance of our results does not imply biochemical significance. Instead, the significant property/volume pairs often suggest further investigations. These investigations may lead to new biochemical insights, or to recognition that the data set was in some way not representative of the population of interest (anomalies caused by sampling errors or improper choice of the control groups). Sometimes the program's findings can be difficult to interpret and we find ourselves going back to the individual structures to see what they mean. This is precisely what the system was designed for. With the radially symmetric calculations, we can look at shells around the central location to understand some of the biophysical interactions that might be critical. We are assessing the utility of using the radial symmetric calculations as a starting point for more advanced studies of the sites.

Our method is not meant as a general solution to the problem of automatic machine induction, but instead is intended as a way to reduce the volume of raw molecular data and present it in a manner that is of use to the investigator. The program is useful for comparing different elements of structure in an exploratory manner. Of course, with sufficient numbers of sites and controls, many of these exploratory investigations may have sufficient statistical significance to be confirmatory as well. In this paper, we have demonstrated the performance for small to moderate numbers of sites (from 6 to $\mathbf{3 0}$ examples). In general, it is easier to find a reasonable set of nonsite examples and to define the nonsite distributions accurately. In those cases when we only have one or two examples of sites, it will be necessary to focus more on the ways in which the sites differ from the (better characterized) nonsites, rather than on a symmetric comparison of the two distributions. At all times, we have a reasonable measure of statistical significance, so we do not fall back on anecdotal descriptions of the important differences.

We have considered the possible effects of the statistical framework on incorrectly reporting results. As detailed in the Methods section, we evaluate significance with the Mann-Whitney rank-sum test. This test is designed to test the null hypothesis that the mean of two arbitrary distributions is different. The test is sensitive to the sample sizes for both sites and nonsites. It is
Table 2. IDs and sample sizes for each of the proteins analyzed

\begin{tabular}{|c|c|c|}
\hline PDB ID ${ }^{a}$ & $\mathrm{~N}$ sites ${ }^{b}$ & $\mathrm{~N}$ nonsites ${ }^{\mathfrak{c}}$ \\
\hline \multicolumn{3}{|c|}{$\begin{array}{c}\text { Calcium binding site } \\
(\text { site }=\text { calcium binding, nonsite }=\text { arbitrary })\end{array}$} \\
\hline $1 \mathrm{NPC}$ & 4 & 20 \\
\hline 1TMN & 4 & 20 \\
\hline 2MSB & 6 & 20 \\
\hline $3 \mathrm{LHM}$ & 1 & 20 \\
\hline 4PTP & 1 & 20 \\
\hline Total & 16 & 100 \\
\hline \multicolumn{3}{|c|}{$\begin{array}{l}\text { Disulfide environment } \\
\text { sulfur, nonsite }=\text { nondisulfide cys sulfur) }\end{array}$} \\
\hline $1 \mathrm{ABE}$ & 0 & 1 \\
\hline 1PAZ & 0 & 1 \\
\hline $1 X Y 1$ & 2 & 0 \\
\hline $2 \mathrm{IG} 2$ & 8 & 2 \\
\hline 2PRK & 4 & 1 \\
\hline $2 \mathrm{SN} 3$ & 8 & 0 \\
\hline $2 \mathrm{TMV}$ & 0 & 1 \\
\hline 3GRS & 2 & 7 \\
\hline 3PSG & 0 & 6 \\
\hline 6PAD & 6 & 1 \\
\hline Total & 30 & 20 \\
\hline \multicolumn{3}{|c|}{ Serine protease active site } \\
\hline $1 \mathrm{ARB}$ & 1 & 5 \\
\hline $1 \mathrm{GCT}$ & 1 & 1 \\
\hline 1SGT & 1 & 0 \\
\hline $1 \mathrm{TON}$ & 1 & 6 \\
\hline $4 \mathrm{PTP}$ & 1 & 2 \\
\hline 8EST & 1 & 5 \\
\hline Total & 6 & 19 \\
\hline
\end{tabular}

\footnotetext{
${ }^{\text {a }}$ Brookhaven PDB identifier.

${ }^{\mathrm{b}}$ Number of sites extracted from the protein.

${ }^{\mathrm{C}}$ Number of nonsites extracted from the protein.
}

possible that two distributions have the same mean, but are very different in shape. In these cases, therefore, we may erroneously label the difference between these distributions as insignificant. We cannot rule out the possibility that some significant differences of this type have been missed. We are confident, however, that the features that are reported as significant by the MannWhitney test are actually significant (each individually) to the level implied by the threshold. For our standard runs with a $P$-value of 0.01 , we expect that approximately $1 \%$ of the features reported are spurious. Thus, our feature plots with an average of 100 significant features may contain approximately one errant result.

We recognize that many of the features we computed are correlated with one another. For example, a shell with an excess of Asp and Glu atoms will have an excess of oxygen atoms as well. We do not count this as a weakness of the approach; there are many alternative ways of understanding the critical features of a site. The set of features we have selected is not in any sense a basis set of features, but is a cognitively useful summary of intermediate level features that simplify the description of these sites. 
As currently implemented, we cannot detect multiple subpopulations within the site or nonsite samples. If these occur, they could be detected by searching for correlations within the feature plots that show dependence of the contents of one property/volume pair on the occurrence of another property/volume pair. The implementation reported here simply reports on marginal distributions of properties and tests for significance of each property/volume pair independently of all other pairs.

Our technique has several features that may make it attractive for exploratory (or confirmatory) analysis of sites within macromolecules. First, it is general purpose. Any environment that is represented as a set of atomic positions can be studied without modifying any of the code. In this paper, we studied three totally different types of sites. Second, the system is modular. The set of properties is easily extended for special purpose analyses. The property calculations are independent of each other, and new properties can be added by writing a small amount of program code. Third, the method analyzes the property distributions within a reasonable statistical framework, with straightforward algorithms. Yet, it relaxes some assumptions that may have limited previous approaches: the control group distributions are not spatially uniform, the choice of controls strongly determines which properties are reported as significant, and the property distributions (for both sites and nonsites) need not be normally distributed. Finally, we have shown that an isotropic (unoriented) analysis of protein microenvironments is useful, even for sites that are known to have oriented structure. The extension of the analysis to include a spatial transformation of the sites into a common coordinate system (either Cartesian or spherical) should provide a more detailed view into those sites.

\section{Methods}

The goal of our method is to characterize the microenvironment of a site. The characterization is expressed using a set of biophysical properties that is evaluated throughout the microenvironment. To avoid characterizing the idiosyncrasies of a single site, a sampling of site instances (all of the same site type) is compared to a set of nonsite instances. The nonsite instances serve as explicit controls against which to assess statistical significance. Our method identifies those properties and spatial volumes in the microenvironment for which there is a statistically significant difference in the distributions of the property values between the site samples and the nonsite samples. The site and nonsite distributions for each property/volume pair are tested independently but plotted together to provide a more global perspective.

The method starts with high-resolution crystal structures as reported in the Protein Data Base (PDB [Bernstein et al., 1977]) that contain examples of the site(s) of interest. The sites are specified by the user as a three-dimensional position and a radius defining the neighborhood of interest. From these structures (or an alternative list of structures), negative examples (control nonsites) are extracted by random sampling, such that they are not within the neighborhood of interest around the site, and such that they contain roughly the same density of atoms. At the user's discretion, nonsites can also be explicitly specified in order to provide a nonrandom control set of data. Given the fragments of the structure files from the PDB that contain sites and nonsites, the algorithm attempts to determine the ways in which the sites and nonsites differ with respect to the distribution of user-defined physical properties.

The algorithmic implementation has, conceptually, four components: (1) a three-dimensional grid for accumulating information about property distributions; (2) a set of property definitions that allow the value of a property at each grid cell to be evaluated; (3) a "collector" that groups grid cells together to form distributions (in the current implementation, the collectors combine grid boxes that are within a shell around the central point of the sites/nonsites ${ }^{1}$; and (4) a method for testing significance. Each will be described in turn.

\section{Grid}

The central spatial representation in the computation is a threedimensional grid that holds the properties of protein's atoms. The grid is cubic, with a unit-cell diagonal chosen to be the length of a carbon-oxygen single bond (giving an edge length of $0.826 \AA$ ), so that two nearby atoms rarely occupy the same cell. The axes of the grid are determined by the coordinate system specified in the PDB file. The value stored in each grid cell is determined by the properties. In the current implementation, only one property value is stored in the grid at a time; the grid storage space is reused.

\section{Properties}

The properties were chosen to span a wide range of biophysical parameters; richness and expressive power guided our choice more than compactness or independence. A complete list of the properties with their definitions is given in Appendix 1. The properties can be grouped roughly into categories based on whether they depend on the identity of the atom, the chemical group to which it belongs, the amino acid to which it belongs, the secondary structure to which it belongs, or some other property calculated on the protein structure. There are many additional properties (or alternative definitions) that can be used, and we selected these because they represent the most common properties associated with routine analysis of binding sites. Each property produces a value for each atom; that value is stored in the grid. Some property values are stored only in the cell that contains the nucleus of the atom; these are marked $\mathrm{NC}$ for nucleus cell. Other properties are distributed over the atom's approximate van der Waals volume and are marked EV (electron volume). To distribute the property value, all cells overlapping a cube, centered on the atom's nucleus, with the same volume as the atom's van der Waals sphere are incremented by the value, scaled by the fraction of the cell/cube overlap. Most of the properties are naturally quantitative, such as charge. Those that are more naturally viewed as categorical (such as atom name or residue name) are reduced to a quantitative form using a 1 to mark instances and 0 to mark noninstances for each element of the category in turn.

\section{Collectors}

Collectors collect the property data in the grid, by summing the values in the grid that fall within the collector's volume. A grid

\footnotetext{
'The system has been built to easily allow other collectors to be built, such as ones that collect grid boxes in such a way that spatial relationships are preserved and not averaged radially.
} 
cell is taken to fall inside a volume if its center point lies inside the volume. Currently the only collection volume used is a shell of thickness $1 \AA$. (We have also experimented with spherical volumes and shell thickness of $2 \AA$ as reported in the sensitivity analysis, and these produce similar results.) Each collector sums the values in consecutive shells of $1 \AA$ thickness (out to a userdefined maximum radius), and returns a vector of summed property values, one value for each collection shell. The collection process is shown graphically in Figure $4 .^{2}$

\section{Testing for significant differences}

The products of the collection stage are site and nonsite distributions. A site distribution for a given property and collection volume contains all the values that were collected for that property/volume pair across all the protein site instances (and thus contains as many values as there are instances). A nonsite distribution is formed analogously. The two distributions are compared for statistical significance. Because these values are not, in general, normally distributed, a nonparametric test (the Mann-Whitney rank-sum test [Glantz, 1987]) is used to compare the distributions to see if the null hypothesis (that the two distributions are the same) can be rejected. All property/volume pairs producing results significant to a user-defined level are displayed in a two-dimensional plot (such as in Figures 1, 2, and 3). The significance level for these experiments was $P<$ 0.01 . Note that although the rank-sum test is invoked many times, each site and nonsite distribution is tested only once, for the property/volume pair from which it was formed and independently from all other possible pairs. The significance level therefore applies to each of those individual tests, not to a global hypothesis about the site microenvironment (no such hypothesis is formed by the system).

The program is written in generic Common Lisp and currently runs on two platforms, Macintosh Common Lisp on the Apple Macintosh, and Hewlett-Packard/Lucid Common Lisp on the Hewlett-Packard 720 series workstation. Those interested in the program code should contact the authors.

The algorithm can be summarized as follows:

INPUT: Set of sites (positive examples), set of nonsites (negative, control examples), set of properties of interest

For each property,

1. Create a grid for site properties

2. For each site,

2.1. Center site on grid; clear grid

2.2. Add value of property for site into grid

2.3. Collect all values within volumes of interest, to produce a list of volume/value pairs giving the site distribution

\footnotetext{
${ }^{2}$ In order to analyze sites in a manner that is sensitive to orientation, one would define collectors that did not perform radial averaging, but kept separate sums of property values for labeled three-dimensional volumes. Such analyses would only make sense if all the sites could be oriented into a common coordinate system before the grids were marked (for example, by defining four common points with which to align the structures). Structures that have not been superimposed and oriented in a common manner require analyses that are radially averaged, as in the experiments reported here.
}

Property: Atom name is $\mathrm{O}$

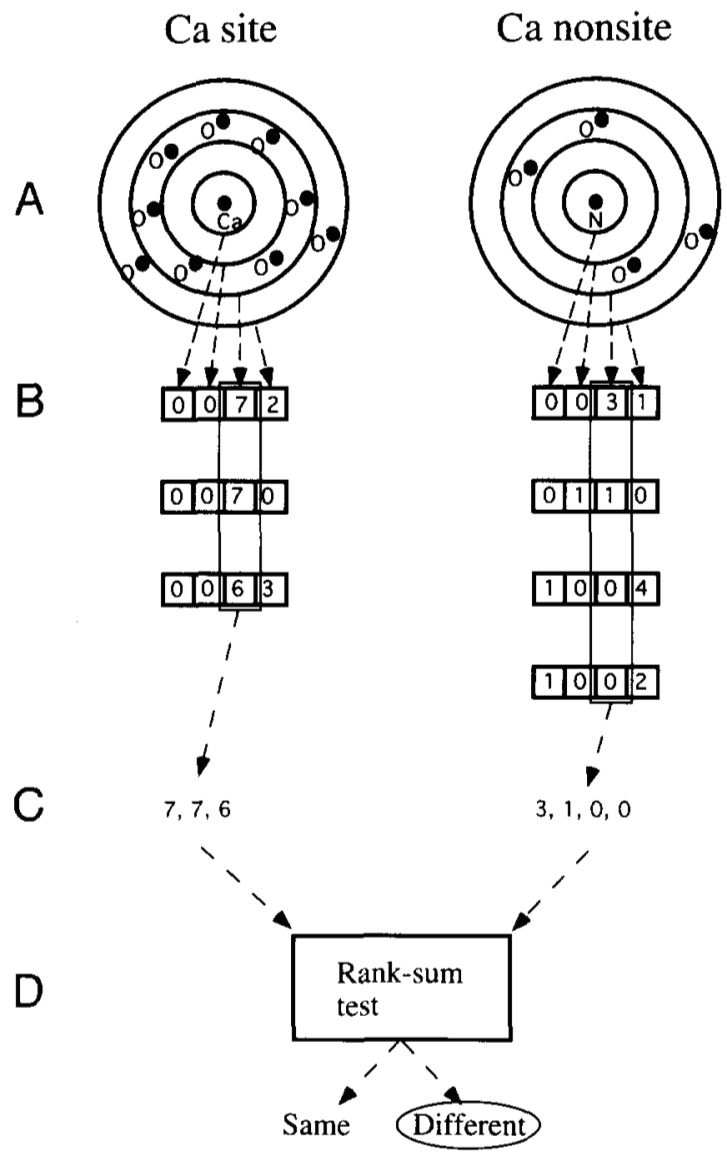

Fig. 4. Summary of procedure used to detect significant features. A representative calcium site and nonsite are shown, in the context of the property "atom name is oxygen." This figure illustrates how the system would conclude that the third shell has significantly more oxygens in calcium sites than in nonsites. A: Shells are formed around each site or nonsite, and values of the property of interest within the grid cells lying in each shell are summed. B: Sums are recorded as a vector, one sum for each shell. C: Values for a property/volume pair (in this example, property $=$ oxygen, volume $=$ shell number 3 ) are collected for all sites to form the site distribution, and analogously over nonsites for the nonsite distribution. D: Site and nonsite distributions are compared using the nonparametric Mann-Whitney rank sum test.

3. Create a grid for nonsite properties

4. For each nonsite,

4.1. Center nonsite on grid; clear grid

4.2. Add value of property for nonsite into grid

4.3. Collect all values within volumes of interest, to produce a list of volume/value pairs giving the nonsite distribution

5. Compare site distribution with nonsite distribution, and report volumes with significant differences for this property.

OUTPUT: List of properties, the volumes in which they show significant differences between sites and nonsites, the magnitude and direction of this difference. 


\section{Application to $\mathrm{Ca}^{2+}$ binding sites}

Calcium $\left(\mathrm{Ca}^{2+}\right)$ is a metal ion commonly bound in proteins. The method was applied to determine which properties correlated with the presence of a calcium binding site. The calcium site was located at the center of the $\mathrm{Ca}^{2+}$ ion, to a radius of $7 \AA$. For a typical binding site, see Figure $5 \mathrm{~A}$. The nonsites were chosen randomly from the same proteins from which the sites were selected, with 20 nonsites per protein. The proteins used and the number of sites and nonsites for each protein are shown in Table 2. Proteins were chosen from lists of commonly studied calcium binding proteins.

\section{Application to disulfide bond sites}

The sulfur atom in a cysteine residue often forms a covalent bond with a sulfur atom in a neighboring cysteine, forming a disulfide bridge. To find the properties correlated with the bonding state of the cysteine, the method was applied, taking the sulfur atom in each cysteine residue as the site's center, out to a radius of $10 \AA$. For cysteines forming a bridge, this will include the other cysteine residue. A typical site is shown in Figure 5B. The control nonsites were chosen to be cysteines not participating in a disulfide bridge (from proteins containing disulfide bridges, as well as some that do not). The proteins used and the number of sites and nonsites for each protein are listed in Table 2. The proteins were chosen at random from the PDB.

\section{Application to serine protease active sites}

Central to the proteolytic activity of serine proteases is presence of a catalytic triad, composed of the side chains from Asp, His, and Ser in a particular three-dimensional organization. The active site does not exhibit radial (spherical) symmetry. A typical active site is shown in Figure 5C. The property search was applied to these sites, using the NE2 atom of the His as the center, to a radius of $10 \AA$. The control nonsites were His residues not in the active site. The proteins used (a selection from the family of serine proteases) and the number of sites and nonsites for each protein are listed in Table 2 .

\section{Sensitivity analysis}

We tested the sensitivity of our results to the choices and assumptions in our method. For each sensitivity test, we changed a parameter (as described below) and then examined the effect on the output representation for changes. We considered four possibilities: a difference between site and control nonsite may have no change, become significant, become insignificant, or reverse significance. (A reversal of significance is the most worrisome situation, because it implies that the parameter is very sensitive to the decision being tested.)

\section{Grid spacing}

The original grid spacing $(0.826 \AA)$ was chosen so that the grid cell diagonal corresponded to the length of a $\mathrm{C}-\mathrm{O}$ bond, which had the effect of producing very few "collisions," when two atoms both fell into the same cell. As a test, the grid spacing was adjusted upward and downward in turn by $20 \%$ (up to $1.00 \AA$, and down to $0.66 \AA$ ), and then all the properties were recomputed for the calcium binding site proteins.

\section{Shell thickness}

The thickness of the collection shells was originally set at $1 \AA$. The calcium binding site proteins were rerun using a shell thickness of $2 \AA$.

\section{van der Waals radii}

Because in reality each atom is not a sphere of fixed radius, we scaled the van der Waals radii used in the property calculations (taken from the standard Richards sets [Richards, 1974], with augmentation from the literature) upward and downward by $20 \%$, followed by a recalculation of all the properties for the calcium binding site proteins.

\section{Choice of nonsite controls}

To highlight the significant effect of how the control group (the nonsites) is chosen, a modification of the serine protease experiment was conducted, using randomly chosen atoms as nonsite centers instead of the NE2 atom in His residues not in the active site.

\section{Size of nonsite sample}

In order to gauge the effects of the sample size, especially on the nonsite group (which we typically have more control over), we compared the results for the calcium binding site run with the number of nonsites reduced by $50 \%$ (from 20 to 10 ). We then ran the analysis again, and compared the output.

\section{Effect of random sampling}

In order to further gauge the effects of sampling for nonsites, we reran the analysis of calcium binding sites with a different random sample of the same number of nonsites $(n=20)$.

\section{Statistical significance cutoff}

Finally, in order to test the sensitivity of our method to the definition of significance, we varied the significance level ( $P$ value). We chose to conduct this experiment on the disulfide and serine protease environments because the results at standard significance level did not include a number of previously described features (as detailed in the Results).

\section{Supplementary material in Electronic Appendix}

The Electronic Appendix (SUPLEMNT directory, Bagley.SUP subdirectory) contains quantitative presentations of the property/volume plots at the standard conditions $(P<0.01$ significance threshold), for the $\mathrm{Ca}$ binding site (first experiment in Fig. 1, file Bagley.ca), disulfide bonding environment (second experiment in Fig. 2, file Bagley.cys), and serine protease active site (second experiment in Fig. 3, file Bagley.his). Each entry contains the significance threshold (the $P$ value), whose sign indicates if the mean value in sites is greater $(+)$ or less than $(-)$ the control group. The rank of the cell is given in parentheses, with (1) being the most significant. The ranks are calculated with full precision of significance values, to break ties.

The Electronic Appendix also contains kinemages of a calcium binding site, a disulfide bridge, and a serine protease active site. 

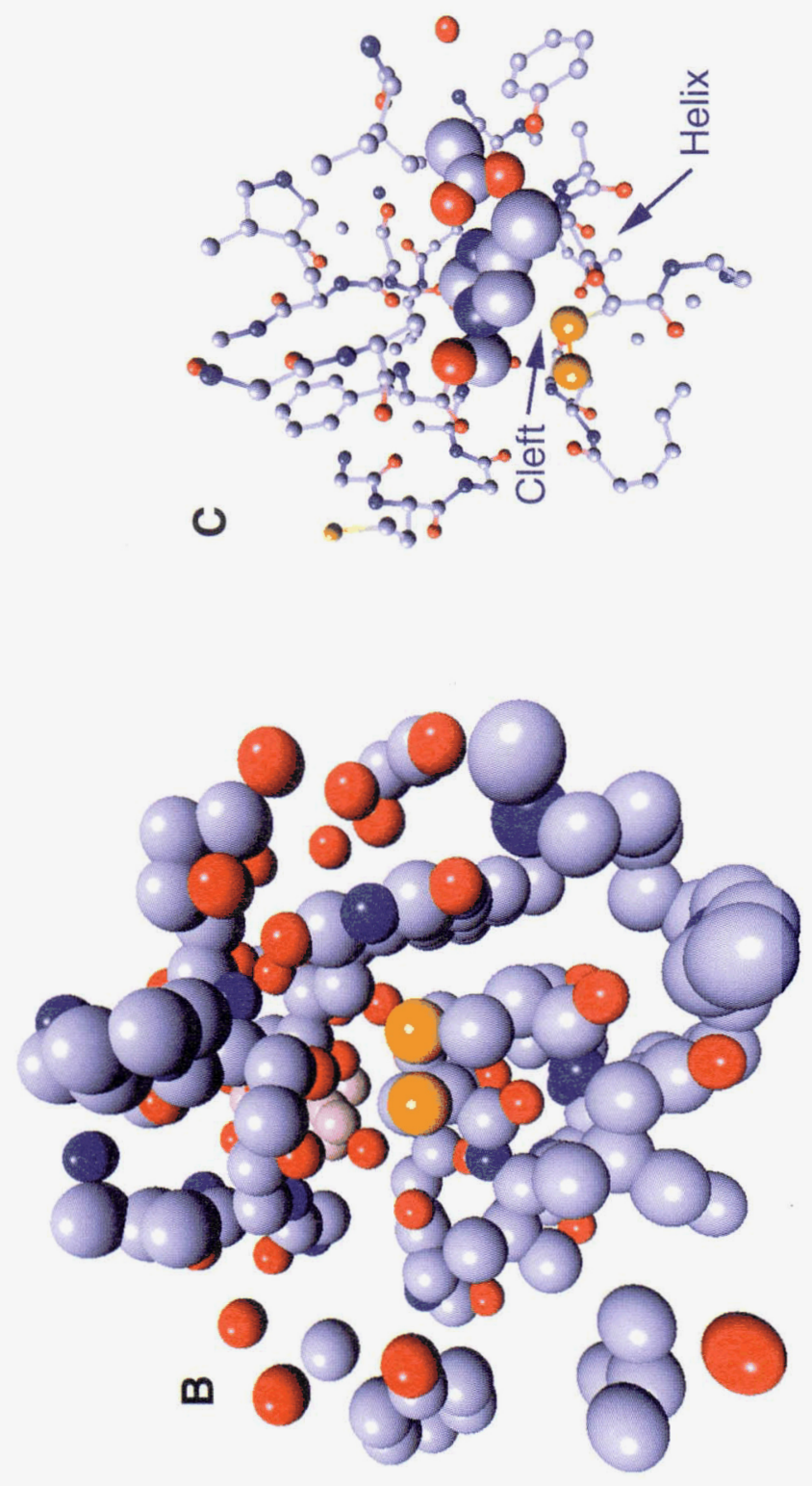

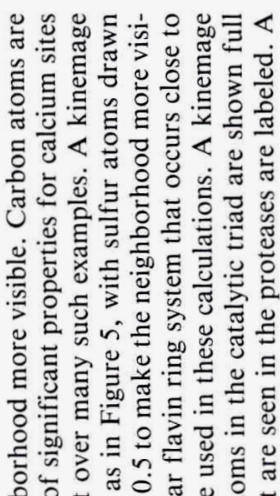

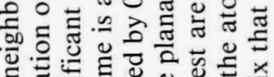

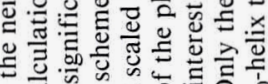

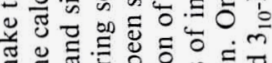

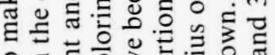

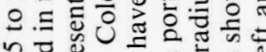

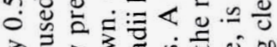

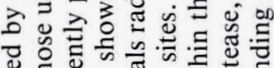

बु

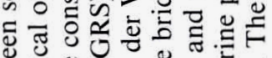

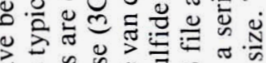

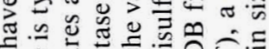

등

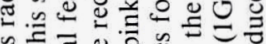

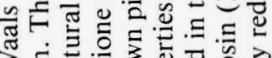

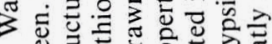

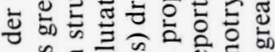

ธ⿻一𠃋十

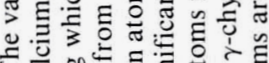

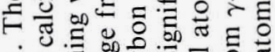

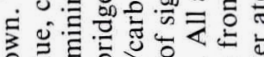

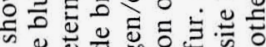

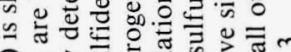

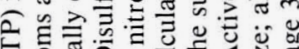

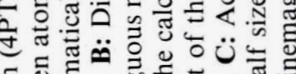

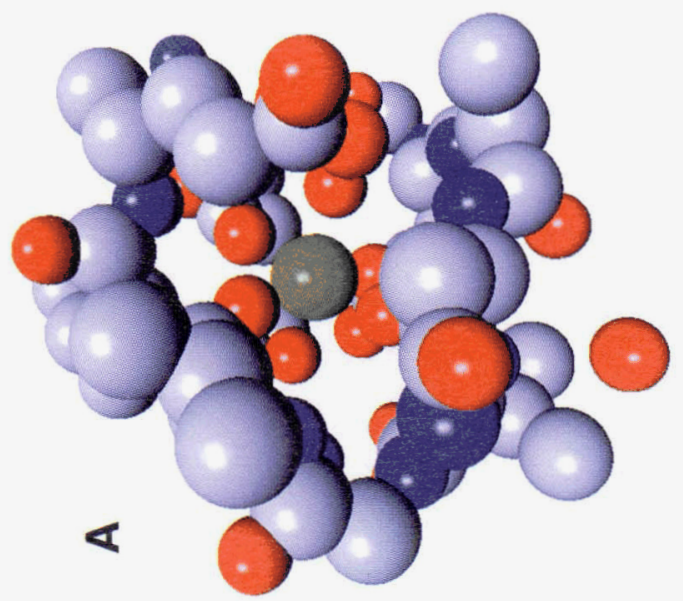

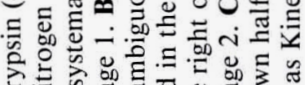

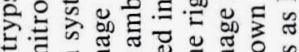

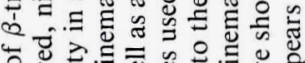

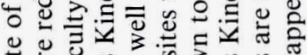

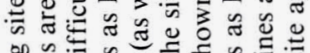

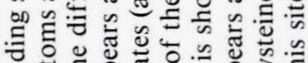

흘

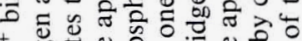

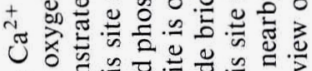

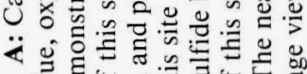

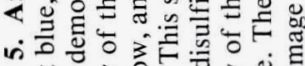

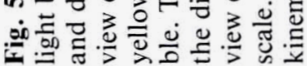




\section{Acknowledgments}

R.B.A. is a Culpeper Foundation Medical scholar and is supported by NIH grant LM-05652. This work was supported by computing resources provided by the Stanford University CAMIS project, which is funded under grant number LM05305.

\section{References}

Barlow DJ, Thornton JM. 1986. The distribution of charged groups in proteins. Biopolymers 25:1717-1733

Bernstein FC, Koetzle TF, Williams GJB, Meyer EF Jr, Brice MD, Rodgers JR, Kennard O, Shimanouchi T, Tasumi M. 1977. The Protein Data Bank: A computer-based archival file for macromolecular structures. $J$ Mol Biol 112:535-542.

Bowie JU, Luthy R, Eisenberg D. 1991. A method to identify protein sequences that fold into a known three-dimensional structure. Science 253:164-170.

Branden C, Tooze J. 1991. Introduction to protein structure. New York: Garland Publishing, Inc.

Chakrabarti P. $1990 \mathrm{a}$. Interaction of metal ions with carboxylic and carboxamide groups in protein structures. Protein Eng 4:49-56.

Chakrabarti P. 1990b. Geometry of interaction of metal ions with histidine residues in protein structures. Protein Eng 4:57-63.

Chakrabarti P. 1993. Anion binding sites in protein structures. $J \mathrm{Mol} \mathrm{Biol}$ $234: 463-482$.

Chakrabarti P. 1994. Conformational analysis of carboxylate and carboxamide side-chains bound to cations. $J$ Mol Biol 239:306-314.

Fiser A, Cserzo M, Tudos E, Simon I. 1992. Different sequence environments of cysteines and half cystines in proteins: Application to predict disulfide forming residues. FEBS Lett 302:117-120.

Glantz SA. 1987. Primer of biostatistics. New York: McGraw-Hill Book Company.

Goodford PJ. 1985. A computational procedure for determining energetically favorable binding sites on biologically important macromolecules. $J$ Med Chem 28:849-857.

Greer J. 1990. Comparative modeling methods: Application to the family of the mammalian serine proteases. Proteins Struct Funct Genet 7:317-334.

Jernigan R, Raghunathan G, Bahar I. 1994. Characterization of interactions and metal ion binding sites in proteins. Curr Opin Struct Biol 4:256-263

Kabsch W, Sander C. 1983. Dictionary of protein secondary structure: Pattern recognition of hydrogen-bonded and geometrical features. Biopolymers $22: 2577-2637$.

Karlin S, Zuker M, Brocchieri L. 1994. Measuring residue associations in protein structures: Possible implications for protein folding. $J \mathrm{Mol} \mathrm{Biol}$ 239:227-248

Klingler TM, Brutlag DL. 1993. Detection of correlations in tRNA sequences with structural implications. First International Conference on Intelligent Systems for Molecular Biology. Menlo Park, California: AAAl Press.

Klingler TM, Brutlag DL. 1994. Discovering structural correlations in $\alpha-$ helices. Protein Sci 3:1847-1857.

Korn AP, Burnett RM. 1991. Distribution and complementarity of hydropathy in multisubunit proteins. Proteins Struct Funct Genet 9:37-55.

Mitchell JBO, Nandi CL, McDonald IK, Thornton JM, Price SL. 1994 Amino/aromatic interactions in proteins: Is the evidence stacked against hydrogen bonding? J Mol Biol 239:315-331.

Muskal SM, Holbrook SR, Kim SH. 1990. Prediction of the disulfidebonding state of cysteine in proteins. Protein Eng 3:667-672.

Nayal M, Di Cera E. 1994. Predicting $\mathrm{Ca}^{2+}$-binding sites in proteins. Proc Natl Acad Sci USA 91:817-821.

Perutz M. 1992. Protein structure: New approaches to disease and therapy. New York: W.H. Freeman and Company.

Reid KSC, Lindley PF, Thornton JM. 1985. Sulphur-aromatic interactions in proteins. FEBS Lett 190:209-213.

Richards FM. 1974. The interpretation of protein structures: Total volume, group volume distributions and packing density. $J$ Mol Biol 82:1-14.

Roe SM, Teeter MM. 1993. Patterns for prediction of hydration around polar residues in proteins. $J$ Mol Biol 229:419-427.

Rooman MJ, Kocher JP, Wodak SJ. 1992. Extracting information on folding from the amino acid sequence: Accurate predictions for protein regions with preferred conformation in the absence of tertiary interactions. Biochemistry $31: 10226-10238$.

Sekharuda YC, Sundaralingam M. 1988. A structure-function relationship for the calcium affinities of regulatory proteins containing "EF-hand" pairs. Protein Eng 2:139-146.

Singh J, Thornton JM. 1992. Atlas of protein side-chain interactions. Oxford: IRL Press.
Sippl MJ. 1990. Calculation of conformational ensembles from potentials of mean force: An approach to the knowledge-based prediction of local structures in globular proteins. $J$ Mol Biol 213:859-883.

Walshaw J, Goodfellow JM. 1993. Distribution of solvent molecules around apolar side-chains in protein crystals. $J$ Mol Biol 231:392-414.

Warme PK, Morgan RS. 1978. A survey of atomic interactions in 21 proteins. J Mol Biol 118:273-287.

Warshel A, Naray-Szabo G, Sussman F, Hwang JK. 1989. How do serine proteases really work? Biochemistry 28:3629-3637.

Yamashita MM, Wesson L, Eisenman G, Eisenberg D. 1990. Where metal ions bind in proteins. Proc Natl Acad Sci USA 87:5648-5652.

Zhou GW, Guo J, Huang W, Fletterick RJ, Scanlan TS. 1994. Crystal structure of a catalytic antibody with a serine protease active site. Science 265:1059-1064.

Zvelebil MJJM, Sternberg MJE, 1988. Analysis and prediction of the location of catalytic residues in enzymes. Protein Eng 2:127-138.

\section{Appendix 1: List of microenvironment properties}

This appendix contains the set of biophysical/biochemical properties currently used by the system. Properties marked $\mathrm{NC}$ are stored only in the cell containing the nucleus of the atom; properties marked EV are spread out over the electron (van der Waals) volume of the atom.

\section{Atom-based properties}

Atom types. One of (ANY, CARBON, NITROGEN, OXYGEN, or OTHER). The atom name is entered in the grid at the location of the atom's nucleus. NC.

Hydrophobicity. All $\mathrm{O}$ and $\mathrm{N}$ are -1 . Any $\mathrm{C}$ directly bonded to an $\mathrm{O}$ or an $\mathrm{N}$ is 0 . All other $\mathrm{C}$ are 0 . All metal ions (Ca, Cu, Fe, $\mathrm{Zn}, \mathrm{Mn}$, $\mathrm{Mg}$ ) are -2 . The $\mathrm{S}$ in Cys is -1 . All other atoms are $0 . \mathrm{EV}$.

Charge. The value is $-1 / 3$ for each of $\mathrm{CG}, \mathrm{OD} 1$, and $\mathrm{OD} 2$ in Asp, $-1 / 3$ for each of CD, OE1, OE2 in Glu, +1 for $N Z$ in Lys, $+1 / 3$ for each of $\mathrm{CZ}, \mathrm{NH} 1, \mathrm{NH} 2$ in $\mathrm{Arg},+2$ for $\mathrm{Ca}, \mathrm{Cu}, \mathrm{Fe}, \mathrm{Mg}, \mathrm{Mn}, \mathrm{Zn}$, and -1 for $\mathrm{Cl}$, and 0 for all other atoms. $\mathrm{EV}$.

Charge-with-His. Similar to charge property, with the addition that His ND1 and His NE2 each are 0.5, and His AD1, His AD2, His AE1, and His AE2 are each 0.25 . EV.

\section{Chemical group-based properties}

Hydroxyl. The value is 1.0 for Ser OG, Thr OG1, or Tyr OH, and 0.5 for Cys SG. 0.0 otherwise. NC

Amide. The value is 1.0 for Asn ND2, Gln NE2, and Pro N, and 0.5 for Arg NH1 and NH2, Asn AD1 and AD2, Gln AEl and AE2, His ND1 and $\mathrm{NE} 2$, and 0.25 for His AD1, AD2, AE1, AE2, 0.0 otherwise. NC,

Amine. The value is 1.0 for Arg NE, Lys NZ, and Trp NE1, 0.5 for Arg NH1 and NH2, and His ND1 and NE2, and 0.25 for His AD1, AD2, AE1, AE2. 0.0 otherwise. NC.

Carbonyl. The value is 1.0 for backbone O, Asn OD1, and GIn OE1 and 0.5 for Asp OD1 and OD2, Asn AD1 and AD2, Gln AE1 and AE2 and Glu OE1 and OE2. 0.0 otherwise. NC.

Ring-system. The value is 1 if the atom is part of a ring system (in His, Phe, Trp, or Tyr). 0 otherwise. NC.

Peptide. The value is 1 if the atom is part of the polypeptide backbone. 0 otherwise. EV.

\section{Residue-based properties}

Residue types. The standard 20 amino acids, or $\mathrm{HOH}$ or Other. NC.

Hydrophobicity classification 1. One of HYDROPHOBIC (Ala, Ile, Leu, Met, Phe, Pro, Val), CHARGED (Arg, Asp, Glu, Lys), POLAR (Asn, Cys, Gln, His, Ser, Thr, Tyr, Trp), or UNKNOWN (nonstandard residues). NC.

Hydrophobicity classification 2. One of NONPOLAR (Ala, Ile, Leu, Met, Phe, Pro, Trp, Val), POLAR (Asn, Cys, Gln, Gly, Ser, Thr, Tyr), ACIDIC (Asp, Glu), or BASIC (Arg, Lys, His), or UNKNOWN (nonstandard residue). $\mathrm{NC}$. 


\section{Secondary structure-based properties}

Secondary structure classification 1. The secondary structure assignment as computed by DSSP (Kabsch \& Sander, 1983), and is one of 3-HELIX, 4-HELIX, 5-HELIX, BRIDGE, STRAND, TURN, BEND, COIL, or HET. NC.

Secondary structure classification 2. A collapsing of the secondary structure classification 1 taxonomy, one of ALPHA (3-helix, 4-helix, 5-helix), BETA (bridge, strand, turn, bend), COIL (coil), or HET (het). NC.

\section{Other properties}

$V D W$-volume, van der Waals volume of the atom. EV.

$B$-factor. $B$-factor recorded for that atom in the PDB. NC.

Mobility. For each residue, the minimum number of bonds to $\mathrm{C} \alpha$ or other backbone atom, with $C \beta$ receiving a value of 1 . NC.

Solvent accessibility. Solvent accessibility of the residue, as computed by DSSP (Kabsch \& Sander, 1983). This is the solvated surface area in units of $10^{2} \AA$. NC. 